\title{
ERG Protein Expression Is of Limited Prognostic Value in Men with Localized Prostate Cancer
}

\author{
Liang Hong Teng, ${ }^{1}$ Cheng Wang, ${ }^{1}$ Michael Dolph, \\ Bryan Donnelly, ${ }^{2,3}$ and Tarek A. Bismar ${ }^{1,3,4,5}$ \\ ${ }^{1}$ Department of Pathology and Laboratory Medicine, University of Calgary and Calgary Laboratory Services, \\ Calgary, AB, Canada T2V $1 P 9$ \\ ${ }^{2}$ Department of Urology, University of Calgary, Calgary, AB, Canada T2N 1N4 \\ ${ }^{3}$ The Prostate Cancer Center, Calgary, AB, Canada T2V $1 P 9$ \\ ${ }^{4}$ Department of Oncology, Biochemistry and Molecular Biology, Calgary, AB, Canada T2N 1N4 \\ ${ }^{5}$ Southern Alberta Cancer Institute and Tom Baker Cancer Center, Calgary, AB, Canada T2N 1N4
}

Correspondence should be addressed to Tarek A. Bismar; tarek.bismar@cls.ab.ca

Received 4 June 2013; Accepted 27 June 2013

Academic Editors: J. H. Ku, T. Nelius, and T. Nishiyama

\begin{abstract}
Copyright (C) 2013 Liang Hong Teng et al. This is an open access article distributed under the Creative Commons Attribution License, which permits unrestricted use, distribution, and reproduction in any medium, provided the original work is properly cited.
\end{abstract}

Background. The prognostic significance of ERG expression in prostate cancer (PCA) has generated mixed results. We sought to investigate the prognostic significance of ERG expression in a localized cohort of men with PCA. Material and Methods. We investigated ERG protein expression in a cohort of 198 men with localized PCA. ERG expression was correlated with patients' clinical outcome and several pathological parameters, including Gleason score (GS), pathological stage, surgical margin, and extracapsular extension. Results. ERG expression was detected in 86/198 (43.4\%) patients exclusively in neoplastic epithelium. Overall, ERG mean expression intensity was $1.01 \pm 1.27$ versus $0.37 \pm 0.83$ in acinar PCA compared to foamy type PCA $(P<0.001)$. In HGPIN, ERG intensity levels were comparable to those in foamy type PCA $(0.13 \pm 0.56)$ but significantly lower than those in acinar PCA $(P<0.001)$. ERG expression was significantly associated with extra-prostatic extension and higher pathological stage and showed a trend toward seminal vesicle invasion. Herein, ERG expression was documented in 50/131 (38.1\%) patients with pT2 versus $30 / 55(54.5 \%)$ patients with pT3 $(P=0.04)$. ERG association with higher pathological stage was more pronounced in patients with GS $>7$. Grouping patients into those with GS $\leq 7$ versus $>7$, there was no significant association between ERG expression and GS. Similarly, no association was present in relation to either surgical margins or postsurgical serum PSA levels. Conclusion. We report significant association between ERG protein levels and extra-prostatic extension and higher pathological stage. ERG expression is not associated with adverse clinical outcome and is of limited prognostic value in localized PCA.

\section{Introduction}

ERG protein expression has been recently suggested to be reflective of ERG gene rearrangements in prostate cancer (PCA) documenting remarkable concordance between the two [1-6]. The rearrangements between the androgen receptor-regulated gene TMPRSS2 (21q22.3) and members of the ETS family member of transcription factor gene, most commonly ERG (21q22.2), are among the most common genetic alterations detected in prostate cancer [7-11]. ERG gene rearrangements have been detected in roughly half
(40-60\%) of PCA of surgical cohorts compared to a rate of $12 \%-15 \%$ in incidental or watchful waiting cohorts $[7,12-18]$.

Previous studies investigating the prognostic significance of $E R G$ gene rearrangements have revealed mixed results [1922]. However, it is becoming more evident that ERG gene rearrangements signify a molecular subtype of PCA.

Some studies investigating the significance of ERG protein expression in localized PCA failed to show association with adverse clinical outcome [23, 24]. However, a recent report by our group demonstrated an association of ERG expression with lethal disease in patients with unsuspected 
and advanced/castrate resistant disease who were treated by transurethral resection of prostate [25]. Moreover, we documented significant association between ERG expression and both Gleason score and tumor volume. Studies from our group and others have also linked ERG status to responsiveness to hormonal therapy, and longer progression time to castration resistant disease, compared to men with no ERG expression $[24,25]$. In the current study, we investigated the association of ERG protein expression to clinicalpathological parameters in a cohort of men with localized prostate cancer.

\section{Material and Methods}

2.1. Study Population and Tissue Microarray Construction. The study cohort consisted of 198 patients who were treated by retropubic radical prostatectomy for localized prostate cancer with a mean followup of 4.8 years (range $0-15.8$ ). Clinical and pathological data were obtained with approval from the institutional review board. Clinical progression was defined as a postoperative serum PSA elevation of $>0.2 \mathrm{ng} / \mathrm{mL}$. Prostate samples were embedded onto three tissue microarray (TMA) blocks using a manual tissue arrayer (Beecher Instruments, Silver Spring, MD, USA). Each block was assembled without prior knowledge of any clinical or pathological staging information. One to nine cores (average $3.3), 0.6 \mathrm{~mm}$ in diameter, were sampled including benign, high grade intraepithelial neoplasia (HGPIN), and prostate cancer (PCA). After construction, $4 \mu \mathrm{m}$ sections were cut and stained with haematoxylin and eosin on the initial slides to verify the histological diagnosis. The study patients' demographics have been previously described [26, 27].

2.2. ERG Protein Expression by Immunohistochemistry (IHC). Briefly, $4 \mu \mathrm{m}$ thick sections from formalin-fixed paraffin-embedded tissue blocks were stained with Ventana autostainer. Prior to the staining, heat-induced antigen retrieval was carried out by vegetable steamer in sodium citrate antigen retrieval buffer $(10 \mathrm{mM} \mathrm{pH} \mathrm{6.0)}$ for 40 minutes and then cooled down to room temperature for about 20 minutes. The slides were incubated for 60 minutes at $37^{\circ} \mathrm{C}$ with ERG rabbit monoclonal antibody (Epitomics, clone EPR 3864) at 1:50 dilution. A Ventana iView DAB detection kit (Ventana Tucson, AZ, USA) was used for HR detection and counter stain.

2.3. Pathological Analysis. The diagnoses of all TMA cores were confirmed by the three study pathologists (LHT, CW, and TAB). Gleason scoring was done according to the 2005 ISUP criteria [28]. For each patient, the two predominant patterns were sampled and included on the TMAs for analysis. ERG protein expression was assessed semiquantitatively using 3-tiered system ( 0 , negative; 1 , low; 2 , high). Cases with either 1 or 2 intensity were considered positive based on previous correlation with ERG gene rearrangement as detected by fluorescent in situ hybridization (data not shown). The ERG antibody was consistently strongly expressed in endothelial
TABLE 1: Demographics of the study patients' cohort.

\begin{tabular}{lccc}
\hline & $\begin{array}{c}\text { ERG negative } \\
\text { (112 patients) }\end{array}$ & $\begin{array}{c}\text { ERG positive } \\
(86 \text { patients) }\end{array}$ & $P$ value \\
\hline Age (years) (mean; range) & $64.31(43-81)$ & $64.00(47-75)$ & 0.735 \\
Pre-PSA level (ng/mL) & & & 0.652 \\
$\leq 10$ & $50(69 \%)$ & $33(73 \%)$ & \\
$>10$ & $22(31 \%)$ & $12(27 \%)$ & \\
Gleason summary & & & 0.521 \\
$<7$ & $34(31 \%)$ & $30(35 \%)$ & \\
7 & $61(55 \%)$ & $47(55 \%)$ & \\
$3+4$ & $36(32 \%)$ & $27(31 \%)$ & \\
$4+3$ & $25(23 \%)$ & $20(24 \%)$ & \\
$>7$ & $16(14 \%)$ & $8(10 \%)$ & \\
pT stage & & & 0.04 \\
pT2 & $81(76 \%)$ & $50(63 \%)$ & \\
pT3 & $25(24 \%)$ & $30(37 \%)$ & \\
Surgical margin & & & 0.886 \\
Negative & $60(57 \%)$ & $45(56 \%)$ & \\
Positive & $46(43 \%)$ & $36(44 \%)$ & \\
\hline
\end{tabular}

cells, which acted as internal control for expression and intensity level.

2.4. Statistical Analysis. Patient characteristics were presented as frequencies and percentages for categorical variables and as means and ranges for continuous variables. Chisquare tests were used to test for associations between ERG protein expression and Gleason score, surgical margin, and pathological stage. The Kaplan-Meier approach along with the log-rank test was used for the survival analyses to test the association between ERG expression and serum PSA relapse. In all statistical tests, a $P$ value $<0.05$ was considered significant.

\section{Results}

3.1. Expression of ERG Protein by Anti-ERG Monoclonal Antibody in Benign, HGPIN, and PCA. Mean patients' age of this cohort was 64 years (range $42.7-80.5$ years) with average follow-up time of 4.8 years (range $0.0-15.8$ months). ERG protein expression was detected in $86 / 198$ patients $(43.4 \%)$. Table 1 demonstrates patients' demographics of the study cohort with respect to ERG expression. Overall, there were no significant differences between the two subgroups (ERG pos \& ERG neg) of patients except for pathological stage, with $37 \%$ of ERG positive tumors detected in pT3 versus $24 \%$ in pT2. To investigate ERG expression in different diagnostic categories, we characterized ERG expression based on individual cores sampled. ERG protein expression was detected in $317 / 788$ (40.2\%) PCA cores. The rate of ERG expression in HGPIN was 4/69 (5.8\%). When we accounted for foamy type PCA morphology, the rate of ERG expression was 15/84 (17.9\%), compared to $302 / 704$ (42.9\%) in acinar PCA (cases with no foamy type morphology) $(P<0.001)$. There was 


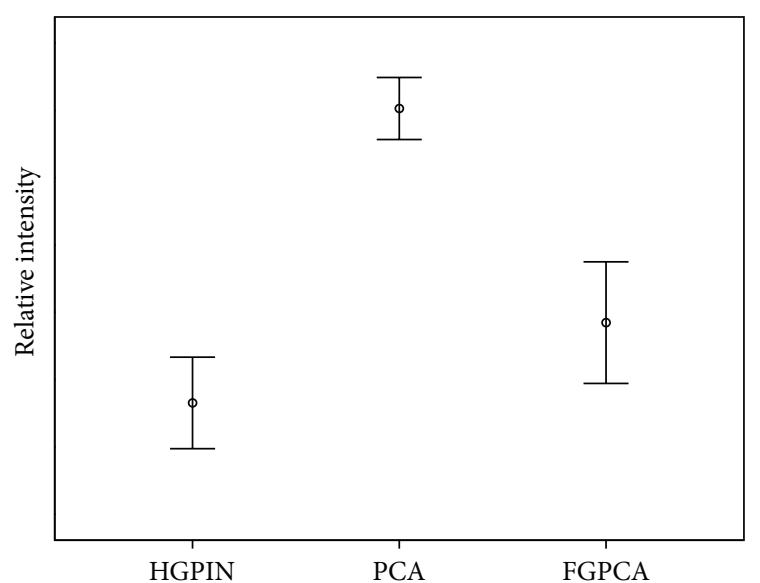

FIGURE 1: Mean intensity level of ERG expression in high grade prostatic intraepithelial neoplasia (HGPIN), foamy gland type prostate cancer (FGPCA), and acinar prostate cancer (PCA). Error bars represent $95 \%$ CI of mean ERG intensity.

no difference in high ERG intensity between foamy type and acinar PCA (data not shown). However, mean intensity level of ERG in acinar PCA was significantly higher than foamy type PCA, $1.01 \pm 1.27$ versus $0.37 \pm 0.83(P<0.001)$. ERG intensity levels in HGPIN were comparable to those in foamy type PCA but slightly lower $(0.13 \pm 0.56)$ but significantly lower than those in acinar PCA $(P<0.001)$ (Figure 1). There were no significant differences in ERG expression between different Gleason scores. ERG expression was noted in 106/280 (37.8\%), $175 / 463$ (37.8\%), and 37/108 (34.2\%) of Gleason scores 6, 7, and 8-10, respectively. Figure 2 demonstrates examples of ERG expression in tissue samples and the distribution of ERG in relation to Gleason score.

\subsection{Expression of ERG in relation to Clinical-Pathological} Parameters and Postsurgical Serum PSA Relapse. When investigating relations between ERG expression and pathological parameters, there was significant association between ERG expression and higher disease stage. In this cohort, ERG expression was present in 50/131 (38.1\%) patients with pT2 versus $30 / 55(54.5 \%)$ patients with pT3 $(P=0.04)$. A similar association was also noted between ERG expression and extra-capsular extension. In this cohort, 52/134 (38.8\%) ERG positive patients demonstrated organ confined disease versus 29/53 (54.7\%) ERG positive patients showing extracapsular extension $(P=0.04)$. Similar trends were noted between ERG expression and seminal vesicle invasion, but this was not statistically significant $(P=0.10)$ (data not shown). No significant association was noted between overall ERG positivity and positive surgical margins (Table 1). Similarly, no association was observed with postsurgical PSA levels when assessed by univariate or multivariate analysis (Figure 3).

Although not informative due to limited patients' numbers, the association between ERG expression and higher stage disease was more pronounced in patients with higher GS. In this cohort, none of the patients with GS $>7$ and ERG
TABLE 2: ERG expression in relation to pathological stage in Gleason score subgroups.

\begin{tabular}{lccc}
\hline ERG expression & \multicolumn{2}{c}{ Path stage } & P value \\
\hline GS $>7$ & pT2 & pT3 & \\
ERG-Neg & $7(100 \%)$ & $8(53 \%)$ & $P(0.02)$ \\
ERG-Pos & $0(0 \%)$ & $7(47 \%)$ & \\
\hline Total & 7 & 15 & \\
\hline GS $<7$ & & & \\
ERG-Neg & $27(56 \%)$ & $2(22 \%)$ & $P(0.06)$ \\
ERG-Pos & $21(44 \%)$ & $7(78 \%)$ & \\
\hline Total & 48 & 9 & \\
\hline
\end{tabular}

positive (0/7) were of stage pT2 compared to $47 \%(7 / 15)$ of ERG negative patients who were of pT2 stage $(P=0.02)$ (Table 2).

\section{Discussion}

This study reports on the potential significance of ERG protein expression in localized prostate cancer. $E R G$ gene rearrangements and ERG expression have been documented in roughly $50 \%$ of localized prostate and locally advanced castrate resistant prostate cancer compared to $12 \%-15 \%$ in watchful waiting or incidental cohorts [16, 17, 22, 25, 29]. Published reports on the significance of ERG expression to patients' outcome are conflicting with some showing association with adverse outcome, while others document no association. Some suggest that it indicates a better prognosis $[16,22,25,29-33]$. This question still needs confirmation in larger studies. However, it is proposed that ERG signifies a molecular class of prostate cancer and may play a role in disease progression within those tumors. This pathway is closely linked to increased rate of PTEN genomic deletions as well as increased ERG expression or ERG gene rearrangements [34, $35]$. ERG gene rearrangements and ERG expression are associated with adverse outcome and lethal disease in watchful waiting or expectant cohorts $[16,25]$. Moreover, patients with ERG overexpression demonstrated shorter progression times to castrate resistance, needing surgical intervention (channel TURP) [25]. In localized prostate cancer, the majority of the reported data suggest no prognostic implication for $E R G$ rearrangements or ERG overexpression in relation to PSA relapse or the recurrence of local disease [22, 36, 37]. However, two earlier reports suggested adverse association of $E R G$ rearrangements with PSA relapse after radical prostatectomy [20, 31]. In Nam's study, ERG status was evaluated using PCR and direct sequencing. Yashimoto's group noted that the adverse prognostic association was linked to PTEN genomic deletions. Of note, a study by Reid et al. documented that $E R G$ gene rearrangements in addition to PTEN genomic deletions had actually favorable outcomes compared to PTEN deletion alone [19]. These observations suggest that the method utilized in determining ERG status, the inclusion or exclusion of other genomic aberration, and the cohort chosen may be the reason for the different results obtained in various studies 


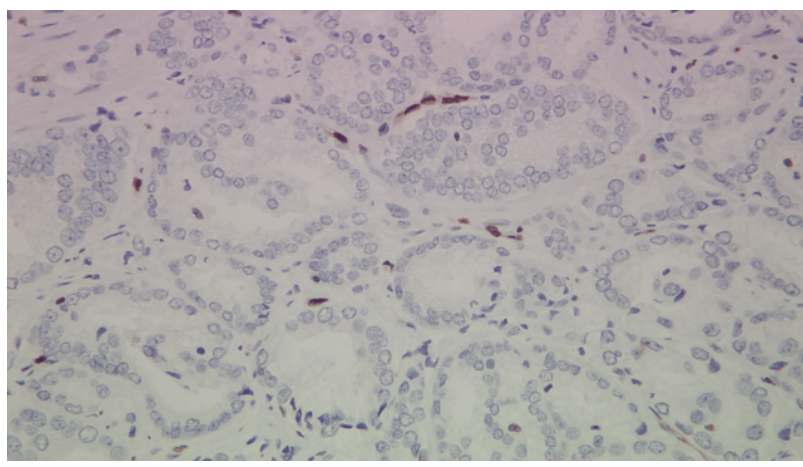

(a)

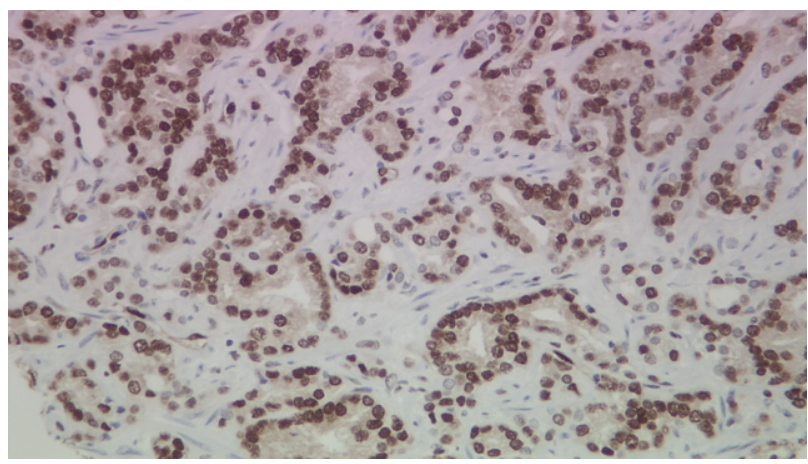

(c)

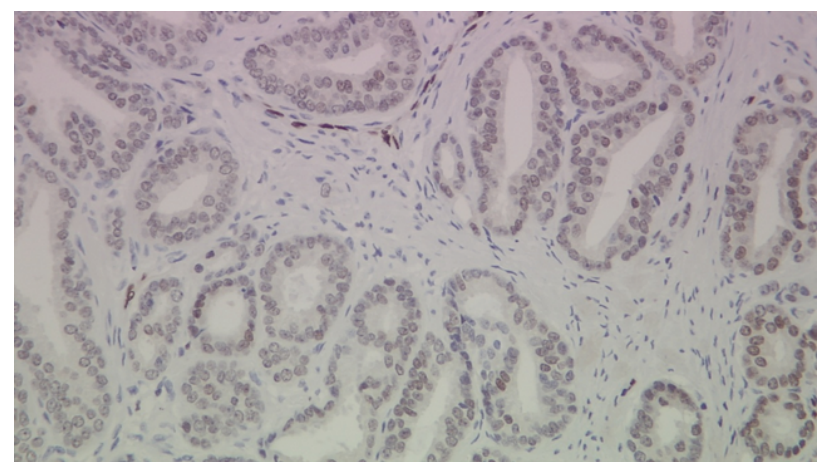

(b)

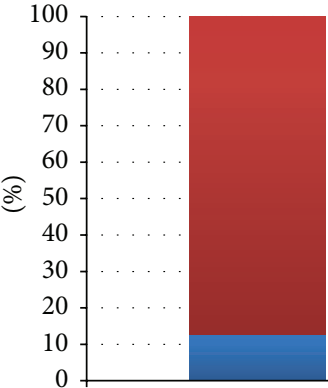

$\mathrm{GS} \leq 7$

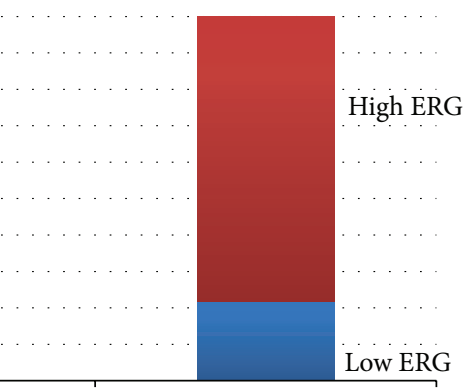

GS $>7$ (d)

FIGURE 2: (a) Prostate cancer Gleason score 6, negative for ERG expression. Note that endothelial cells acting as positive control show strong ERG expression. (b) Prostate cancer Gleason score 6, showing weak intensity ERG expression. (c) Prostate cancer Gleason score 7 (3 + 4), showing high intensity ERG expression. (d) ERG low and high intensity distribution percentages in patients with GS $\leq 7$ versus $>7$.

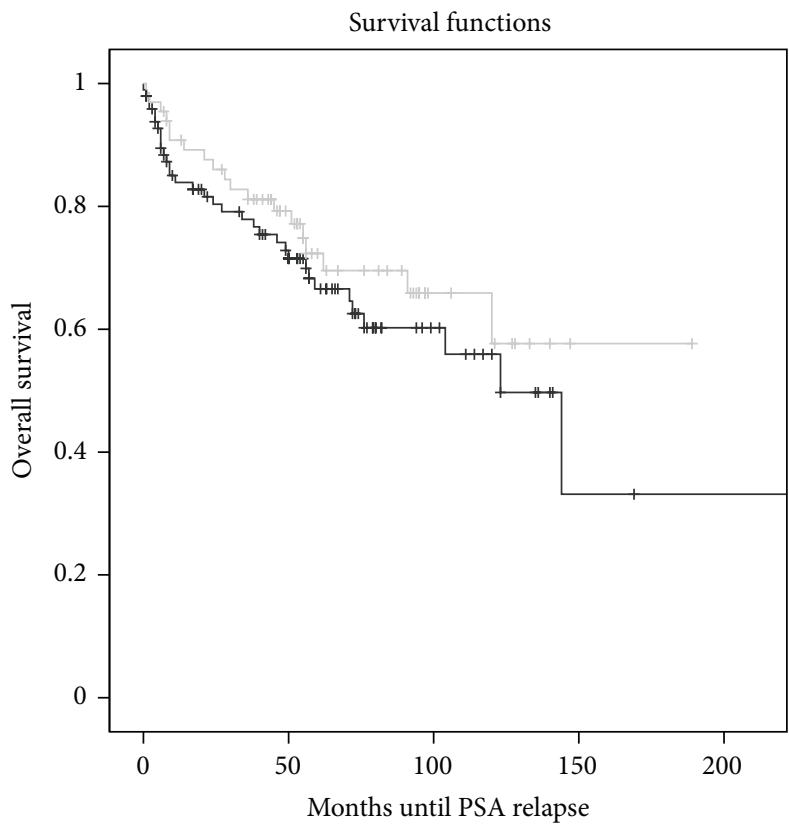

ERG

$\neg$ Negative ERG expression

$\square$ Positive ERG expression

+ Negative ERG expression-censored

$\longrightarrow$ Positive ERG expression-censored

FIGURE 3: Kaplan-Meier curve of association between ERG expression and PSA relapse after radical prostatectomy $(P=0.31)$. 
investigating the role of ERG in prostate cancer. Our results support previous observations showing no prognostic relationship between ERG overexpression and clinical outcome in localized prostate cancer. However, in contrast to earlier studies, we document significant association between ERG expression and some pathological parameters. In this cohort, patients whose tumors were ERG positive were at higher risk of exhibiting extra-prostatic extension and increased disease stage compared to patients whose tumors did not express ERG. Specifically, all patients with GS $>7$ and ERG expression showed pT3 stage disease compared to none of pT2. However, this data is limited by the number of patients within each GS subgroup and need further confirmation. More importantly, in addition to not documenting any clinical prognostic significance for ERG expression, we did not observe any association between ERG expression and other pathological parameters such as Gleason score or surgical margins, which further diminishes any potential prognostic significance for ERG expression, at least in localized PCA.

Another issue worth mentioning regarding ERG is that, $E R G$ gene rearrangements have been previously associated with specific histopathological features and detected more frequently in some morphologic variants of prostate cancer than others [38]. In this study, we confirm lower rates of ERG expression in tumors with foamy/xanthomatous morphology which demonstrated lower mean expression intensities compared to acinar PCA. The significance of this is not yet known, but suggests that those two types of tumors may be different at the molecular level.

In conclusion, this study demonstrates significant association between ERG expression and extra-prostatic extension and higher pathological stage in localized prostate cancer. It also demonstrates no prognostic correlation of ERG expression with patients' clinical outcome. Moreover, the lack of association with any other pathological parameters also significantly diminishes any potential clinical application of ERG expression, at least in men with localized prostate cancer.

\section{Conflict of Interests}

The authors have no conflict of interests to declare in this study.

\section{Acknowledgments}

This work was supported by the Young Investigator Award of the Prostate Cancer Foundation, USA. Prostate Cancer Canada, and is proudly funded by theMovember foundation, Grant no. 132013-01.

\section{References}

[1] G. J. van Leenders, J. L. Boormans, C. J. Vissers et al., "Antibody EPR3864 is specific for ERG genomic fusions in prostate cancer: implications for pathological practice," Modern Pathology, vol. 24, no. 8, pp. 1128-1138, 2011.
[2] B. Furusato, S. H. Tan, D. Young et al., "ERG oncoprotein expression in prostate cancer: clonal progression of ERGpositive tumor cells and potential for ERG-based stratification," Prostate Cancer and Prostatic Diseases, vol. 13, no. 3, pp. 228-237, 2010.

[3] T. L. Lotan, N. S. Gupta, W. Wang et al., "ERG gene rearrangements are common in prostatic small cell carcinomas," Modern Pathology, vol. 24, no. 6, pp. 820-828, 2011.

[4] M. Miettinen, Z. F. Wang, A. Paetau et al., "ERG transcription factor as an immunohistochemical marker for vascular endothelial tumors and prostatic carcinoma," The American Journal of Surgical Pathology, vol. 35, no. 3, pp. 432-441, 2011.

[5] K. Park, S. A. Tomlins, K. M. Mudaliar et al., "Antibody-based detection of ERG rearrangement-positive prostate cancer," Neoplasia, vol. 12, no. 7, pp. 590-598, 2010.

[6] A. Chaux, R. Albadine, A. Toubaji et al., "Immunohistochemistry for ERG expression as a surrogate for TMPRSS2-ERG fusion detection in prostatic adenocarcinomas," The American Journal of Surgical Pathology, vol. 35, no. 7, pp. 1014-1020, 2011.

[7] S. A. Tomlins, D. R. Rhodes, S. Perner et al., "Recurrent fusion of TMPRSS2 and ETS transcription factor genes in prostate cancer," Science, vol. 310, no. 5748, pp. 644-648, 2005.

[8] M. J. Soller, M. Isaksson, P. Elfving, W. Soller, R. Lundgren, and I. Panagopoulos, "Confirmation of the high frequency of the TMPRSS2/ERG fusion gene in prostate cancer," Genes Chromosomes and Cancer, vol. 45, no. 7, pp. 717-719, 2006.

[9] S. A. Tomlins, R. Mehra, D. R. Rhodes et al., “TMPRSS2:ETV4 gene fusions define a third molecular subtype of prostate cancer," Cancer Research, vol. 66, no. 7, pp. 3396-3400, 2006.

[10] B. E. Helgeson, S. A. Tomlins, N. Shah et al., "Characterization of TMPRSS2:ETV5 and SLC45A3:ETV5 gene fusions in prostate cancer," Cancer Research, vol. 68, no. 1, pp. 73-80, 2008.

[11] K. G. Hermans, H. A. van der Korput, R. van Marion et al., "Truncated ETV1, fused to novel tissue-specific genes, and fulllength ETV1 in prostate cancer," Cancer Research, vol. 68, no. 18, pp. 7541-7549, 2008.

[12] S. Perner, F. Demichelis, R. Beroukhim et al., "TMPRSS2:ERG fusion-associated deletions provide insight into the heterogeneity of prostate cancer," Cancer Research, vol. 66, no. 17, pp. 83378341, 2006.

[13] W. Liu, C. M. Ewing, B. L. Chang et al., "Multiple genomic alterations on 21q22 predict various TMPRSS2/ERG fusion transcripts in human prostate cancers," Genes Chromosomes and Cancer, vol. 46, no. 11, pp. 972-980, 2007.

[14] L. M. FitzGerald, I. Agalliu, K. Johnson et al., "Association of TMPRSS2-ERG gene fusion with clinical characteristics and outcomes: results from a population-based study of prostate cancer," BMC Cancer, vol. 8, article 230, 2008.

[15] D. Hessels, F. P. Smit, G. W. Verhaegh, J. A. Witjes, E. B. Cornel, and J. A. Schalken, "Detection of TMPRSS2-ERG fusion transcripts and prostate cancer antigen 3 in urinary sediments may improve diagnosis of prostate cancer," Clinical Cancer Research, vol. 13, no. 17, pp. 5103-5108, 2007.

[16] F. Demichelis, K. Fall, S. Perner et al., "TMPRSS2:ERG gene fusion associated with lethal prostate cancer in a watchful waiting cohort," Oncogene, vol. 26, no. 31, pp. 4596-4599, 2007.

[17] S. Liu, M. Yoshimoto, K. Trpkov et al., "Detection of ERG gene rearrangements and PTEN deletions in unsuspected prostate cancer of the transition zone," Cancer Biology and Therapy, vol. 11, no. 6, pp. 562-566, 2011. 
[18] T. L. Lotan, A. Toubaji, R. Albadine et al., "TMPRSS2-ERG gene fusions are infrequent in prostatic ductal adenocarcinomas," Modern Pathology, vol. 22, no. 3, pp. 359-365, 2009.

[19] A. H. M. Reid, G. Attard, L. Ambroisine et al., "Molecular characterisation of ERG, ETV1 and PTEN gene loci identifies patients at low and high risk of death from prostate cancer," The British Journal of Cancer, vol. 102, no. 4, pp. 678-684, 2010.

[20] R. K. Nam, L. Sugar, W. Yang et al., "Expression of the TMPRSS2:ERG fusion gene predicts cancer recurrence after surgery for localised prostate cancer," The British Journal of Cancer, vol. 97, no. 12, pp. 1690-1695, 2007.

[21] O. R. Saramäki, A. E. Harjula, P. M. Martikainen, R. L. Vessella, T. L. J. Tammela, and T. Visakorpi, "TMPRSS2.ERG fusion identifies a subgroup of prostate cancers with a favorable prognosis," Clinical Cancer Research, vol. 14, no. 11, pp. 33953400, 2008.

[22] A. D. Darnel, C. J. LaFargue, R. T. Vollmer, J. Corcos, and T. A. Bismar, "TMPRSS2-ERG fusion is frequently observed in gleason pattern 3 prostate cancer in a canadian cohort," Cancer Biology and Therapy, vol. 8, no. 2, pp. 125-130, 2009.

[23] A. M. Hoogland, G. Jenster, W. M. van Weerden et al., "ERG immunohistochemistry is not predictive for PSA recurrence, local recurrence or overall survival after radical prostatectomy for prostate cancer," Modern Pathology, vol. 25, no. 3, pp. 471479, 2012.

[24] S. Minner, M. Enodien, H. Sirma et al., "ERG status is unrelated to PSA recurrence in radically operated prostate cancer in the absence of antihormonal therapy," Clinical Cancer Research, vol. 17, no. 18, pp. 5878-5888, 2011.

[25] T. A. Bismar, M. Dolph, L. H. Teng, S. Liu, and B. Donnelly, "ERG protein expression reflects hormonal treatment response and is associated with Gleason score and prostate cancer specific mortality," European Journal of Cancer, vol. 48, no. 4, pp. 538546, 2012.

[26] A. D. Darnel, E. Behmoaram, R. T. Vollmer et al., "Fascin regulates prostate cancer cell invasion and is associated with metastasis and biochemical failure in prostate cancer," Clinical Cancer Research, vol. 15, no. 4, pp. 1376-1383, 2009.

[27] F. Brimo, R. T. Vollmer, M. Friszt, J. Corcos, and T. A. Bismar, "Syndecan-1 expression in prostate cancer and its value as biomarker for disease progression," The British Journal of Urology International, vol. 106, no. 3, pp. 418-423, 2010.

[28] J. I. Epstein, W. C. Allsbrook Jr., M. B. Amin et al., "The 2005 international society of urological pathology (ISUP) consensus conference on Gleason grading of prostatic carcinoma," The American Journal of Surgical Pathology, vol. 29, no. 9, pp. 12281242, 2005.

[29] S. Perner, J. Mosquera, F. Demichelis et al., "TMPRSS2-ERG fusion prostate cancer: an early molecular event associated with invasion," The American Journal of Surgical Pathology, vol. 31, no. 6, pp. 882-888, 2007.

[30] O. R. Saramäki, A. E. Harjula, P. M. Martikainen, R. L. Vessella, T. L. J. Tammela, and T. Visakorpi, "TMPRSS2.ERG fusion identifies a subgroup of prostate cancers with a favorable prognosis," Clinical Cancer Research, vol. 14, no. 11, pp. 33953400, 2008.

[31] M. Yoshimoto, A. M. Joshua, I. W. Cunha et al., "Absence of TMPRSS2:ERG fusions and PTEN losses in prostate cancer is associated with a favorable outcome," Modern Pathology, vol. 21, no. 12, pp. 1451-1460, 2008.

[32] A. Gopalan, M. A. Leversha, J. M. Satagopan et al., “Tmprss2-erg gene fusion is not associated with outcome in patients treated by prostatectomy," Cancer Research, vol. 69, no. 4, pp. 1400-1406, 2009.

[33] G. Attard, J. S. de Bono, J. Clark, and C. S. Cooper, "Studies of TMPRSS2-ERG gene fusions in diagnostic trans-rectal prostate biopsies," Clinical Cancer Research, vol. 16, no. 4, p. 1340, 2010.

[34] B. Han, R. Mehra, R. J. Lonigro et al., "Fluorescence in situ hybridization study shows association of PTEN deletion with ERG rearrangement during prostate cancer progression," Modern Pathology, vol. 22, no. 8, pp. 1083-1093, 2009.

[35] T. A. Bismar, M. Yoshimoto, R. T. Vollmer et al., "PTEN genomic deletion is an early event associated with ERG gene rearrangements in prostate cancer," The British Journal of Urology International, vol. 107, no. 3, pp. 477-485, 2011.

[36] K. G. Hermans, J. L. Boormans, D. Gasi et al., "Overexpression of prostate-specific TMPRSS2(exon 0)-ERG fusion transcripts corresponds with favorable prognosis of prostate cancer," Clinical Cancer Research, vol. 15, no. 20, pp. 6398-6403, 2009.

[37] A. M. Hoogland, G. Jenster, W. M. van Weerden et al., "ERG immunohistochemistry is not predictive for PSA recurrence, local recurrence or overall survival after radical prostatectomy for prostate cancer," Modern Pathology, vol. 25, no. 3, pp. 471479, 2012.

[38] J. M. Mosquera, S. Perner, F. Demichelis et al., "Morphological features of TMPRSS2-ERG gene fusion prostate cancer," Journal of Pathology, vol. 212, no. 1, pp. 91-101, 2007. 


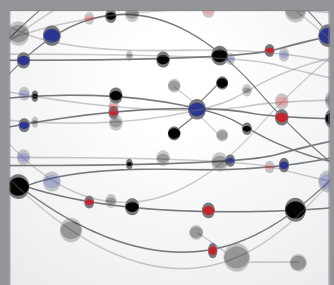

The Scientific World Journal
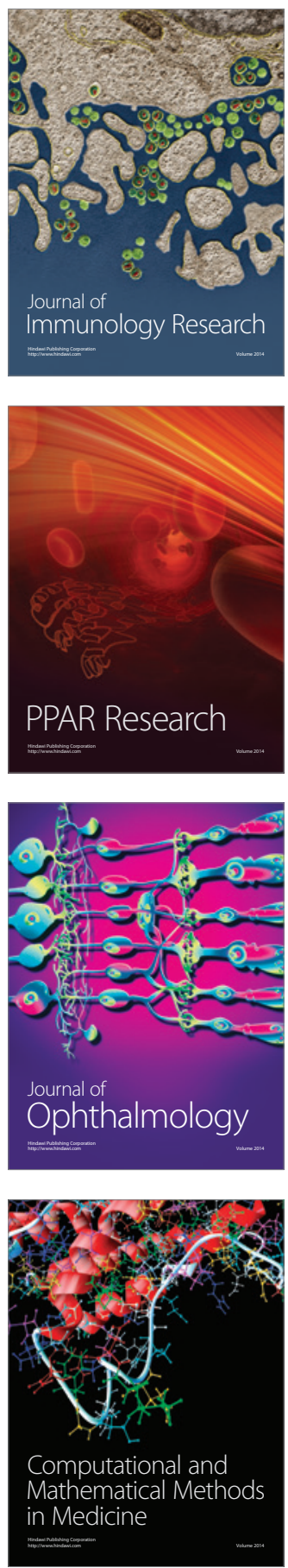

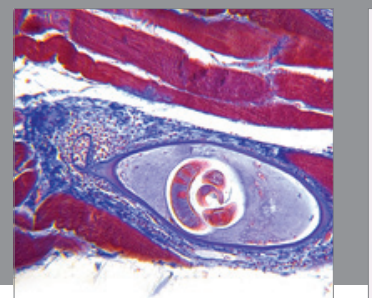

Gastroenterology

Research and Practice
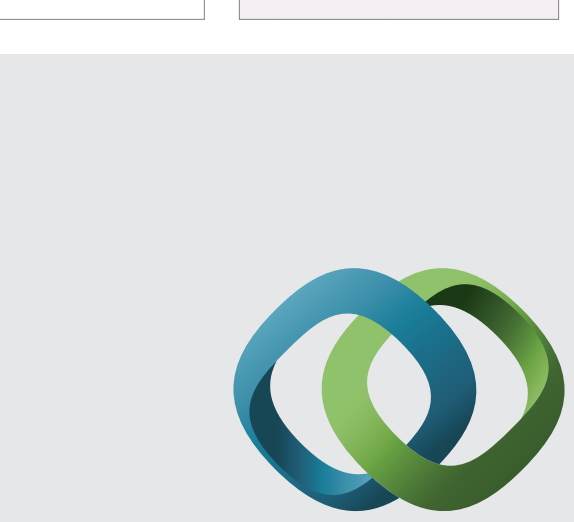

\section{Hindawi}

Submit your manuscripts at

http://www.hindawi.com
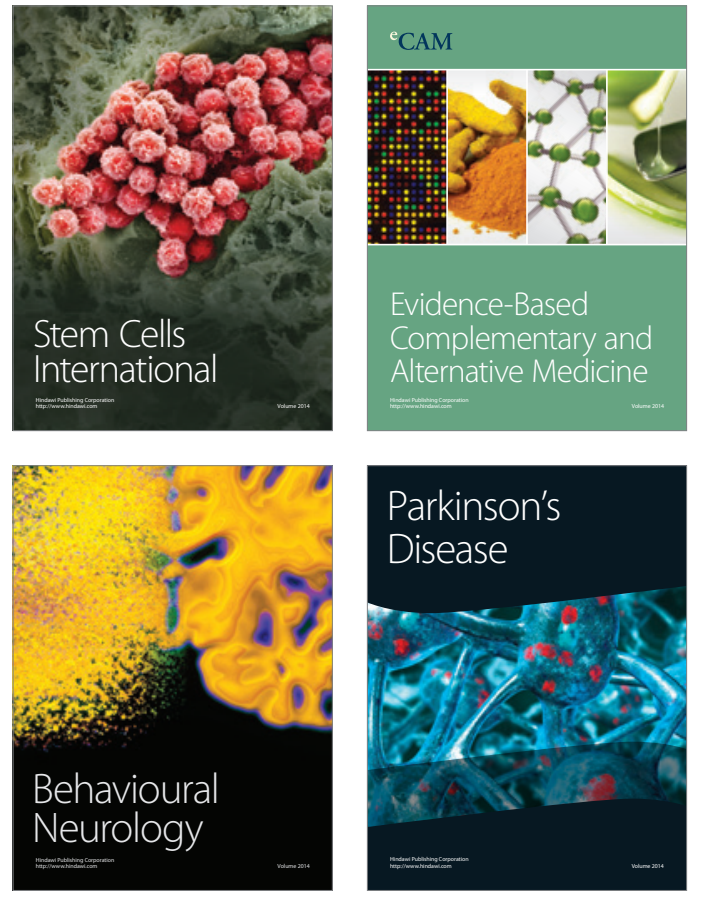
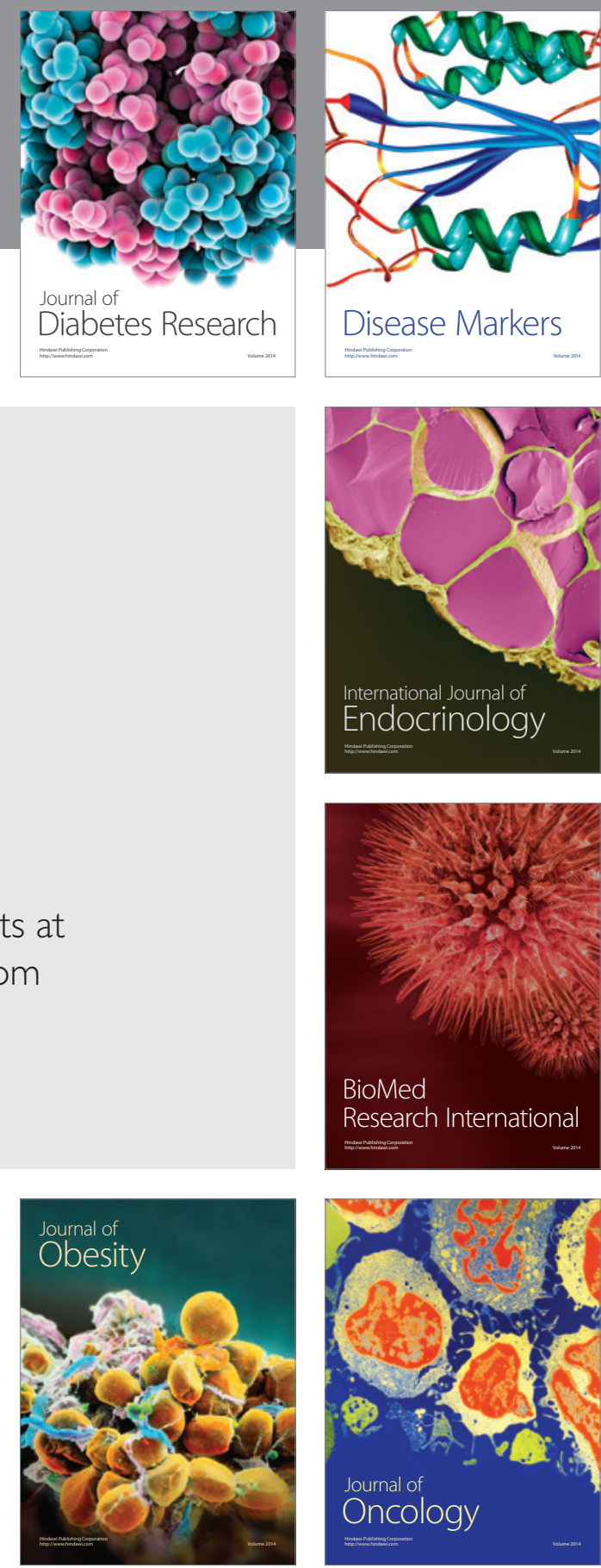

Disease Markers
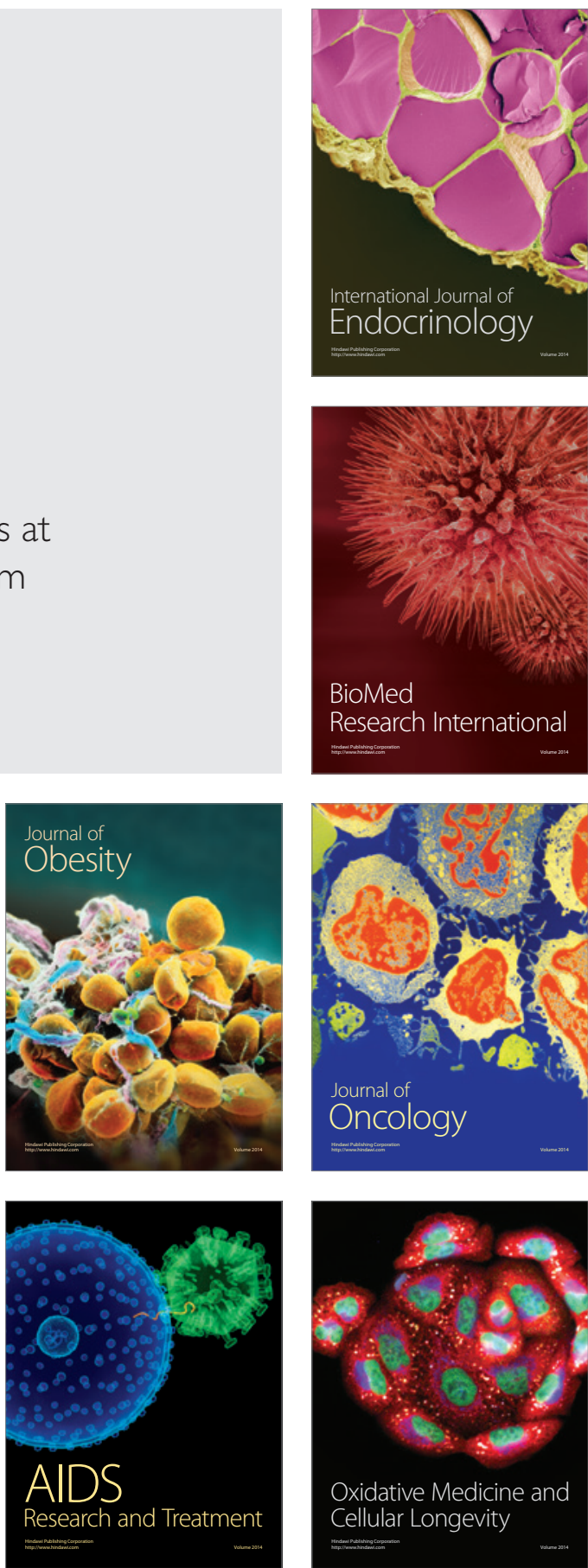